\title{
Virtual residency/fellowship interviews: The good, the bad, and the future
}

\author{
Patrick A. Ho, MD, MPH
}

A $s$ a psychiatry resident in the age of coronavirus disease 2019 (COVID19), many of my educational experiences have undergone adjustments. Now, as I interview for a fellowship, I see firsthand that recruitment activities have not been spared from shifting paradigms levied by the pandemic. To adhere to social distancing guidelines and limit trainee interpersonal exposure, the American Association of Directors of Psychiatric Residency Training recommended that all psychiatry residency interviews be conducted virtually for 2020/2021. ${ }^{1}$ Trainees and programs alike are embarking on a new frontier of virtual interviews, and it is important that we evaluate the advantages and disadvantages of this approach. Because uncertainty abounds regarding when a sense of normalcy might eventually return to psychiatry residency and fellowship recruitment activities, I also provide recommendations to interviewers and interviewees who may navigate virtual recruitment in the future.

\section{Advantages of virtual interviews}

An immediately significant advantage of virtual interviews is the lack of travel, which for some applicants can be cost-prohibitive. The costs of airfare, rental vehicles, and lodging in multiple cities can add up, sometimes requiring students to budget interview travel into already-high student loans. In some cases, applicants may have limited days to interview, which makes the flexibility afforded by the lack of travel

advantageous. Furthermore, navigating new locations can add to preexisting interview stress. Without travel, applicants can consider a broader set of programs and accept more interviews.

Another advantage is that virtual interviews allow interviewees latitude to shift the interview's "frame." Rather than sitting in an interviewer's office, interviewees can choose a more comfortable environment for themselves, imparting a "home-field advantage" that may put them at ease. During my fellowship interviews, controlling the room temperature, using a familiar chair, and wearing comfortable shoes helped to reduce the anxiety inherent to interviewing.

\section{Disadvantages of virtual interviews}

Any new or unfamiliar experience can impart challenges. For example, applicants and interviewers must adjust to and observe different sets of etiquette during virtual interviews. These include muting microphones to avoid talking over each other, maintaining consistent eye contact, avoiding multitasking, and following up to avoid miscommunication.

continued

LET YOUR VOICE BE HEARD

CURRENT PSYCHIATRY invites psychiatry residents to share their views on professional or clinical topics for publication in Residents' Voices. E-mail jbauer@mdedge.com for author guidelines.

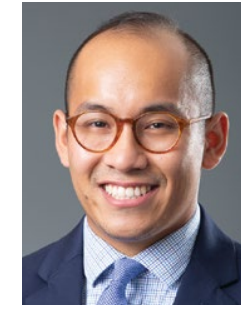

Dr. Ho is a PGY-6 Resident, Psychiatry and Preventive Medicine, Dartmouth-Hitchcock Medical Center, Lebanon, New Hampshire. Disclosure

The author reports no financial relationships with any companies whose products are mentioned in this article, or with manufacturers of competing products.

doi: $10.12788 / c p .0075$ 


\section{Clinical Point}

\section{Both applicants and interviewers must observe different sets of etiquette during virtual interviews}

\title{
Table
}

\section{Recommendations for applicants and psychiatry programs participating in virtual interviews}

\author{
Recommendations for applicants \\ - dress as you would for an in-person interview, including professional but comfortable clothing \\ and footwear \\ - make the "frame" of your interview comfortable yet professional by selecting the optimal room \\ temperature, seating, background, and lighting \\ - practice using the selected virtual platform in order to assess the internet connection and become \\ comfortable using the platform controls and settings \\ - attempt to assess a program's culture by asking questions about interviewers' experiences and \\ their favorite/least favorite features of the program \\ Recommendations for psychiatry programs \\ - schedule breaks between interviews to prevent interviewee (and interviewer) fatigue \\ - keep interviews limited to 1 day if possible \\ - make interviews accessible for applicants in all time zones \\ - practice with "mock interviews" to help interviewers prepare, assess for technical problems, and \\ gauge the virtual interview flow \\ - use innovative ways to display your program's culture, such as by hosting a virtual "happy hour" \\ with current residents or having multiple interviewers with an applicant to allow them to observe \\ faculty interactions
}

Another potential problem is that virtual interviews can dampen an applicant's ability to appreciate a program's culture. Observing informal interactions between trainees and faculty is often as important as the formal interviews in ascertaining which programs have a supportive culture. Because my virtual fellowship interviews have generally been limited to formal one-on-one interviews, assessing program culture has become more challenging. Conversely, programs may find it difficult to grasp an applicant's temperament and interaction style.

Virtual interviewing, while undeniably convenient in many regards, may fall prey to its own convenience. There can be disparities in the quantity, duration, and frequency of interviews. For me, the number of and time allotted for interviews has varied widely, ranging from 2.5 to 8 hours. The amount of allotted break time has also differed, with some programs providing longer breaks between interviews (30 to 60 minutes) and others offering shorter (5 to 10 minutes) or no breaks. Minimal breaks may fatigue applicants, while longer breaks may seem like wasted time. While virtual interviews may require no physical travel between offices, breaks are a necessity that should be implemented thoughtfully.

Finally, a troublesome challenge I encountered surprisingly often was unreliable internet service and other technical difficulties. Several times, my interviewers' (or my) screen froze or shut off due to connectivity issues. This is an obstacle unique to virtual interviews that requires both a backup plan as well as patience and calm to navigate during an already taxing situation.

\section{What's next?}

As applicants and programs adjust to the realities of virtual interviewing, this is likely an unfamiliar experience for all. While the benefits and shortcomings must be considered together, I, along with many of my peers, ${ }^{2}$ continue to prefer traditional in-person interviews. As the ongoing COVID-19 pandemic makes inperson interviews difficult, applicants and programs must embrace the experience 
of virtual interviews. However, a good understanding of the advantages and disadvantages are instrumental in preempting prospective challenges. Based on my recent experiences with virtual fellowship interviews, I have created some recommendations for applicants and psychiatry programs participating in virtual recruitment (Table, page e12).

After the COVID-19 pandemic subsides, it is conceivable that the advantages of virtual interviewing may justify its continued use. For example, applicants may be able to apply to geographically diverse programs without travel expenses. Currently, there is a paucity of evidence regarding virtual interviews specifically in psychiatry training programs, but the experiences of both applicants and psychiatry programs during this atypical time will allow us to improve the process going forward, and evaluate its utility well after COVID-19 recedes.

\section{References}

1. Arbuckle M, Kerlek A, Kovach J, et al. Consensus statement from the Association of Directors of Medical Student Education in Psychiatry (ADMSEP) and the American Association of Directors of Psychiatric Residency Training (AADPRT) on the 2020-21 Residency Application Cycle. https://www.aadprt. org/application/files/8816/0017/8240/admsep_aadprt_ statement_9-14-20_Rev.pdf. Published May 18, 2020. Accessed November 20, 2020.

2. Seifi A, Mirahmadizadeh A, Eslami V. Perception of medical students and residents about virtual interviews for residency applications in the United States. PLoS ONE. 2020; 15(8):e0238239. doi: 10.1371/journal.pone.0238239.

\section{Clinical Point}

After COVID-19, the advantages of virtual interviewing may justify its continued use 